incomplete resection rates at colonoscopy was noted for such polyps at local units. We hence established a National Clinical Network of specialist expert advisors, meeting every fortnight via videoconference to review endoscopy, radiology, pathology and clinical data of cases referred through specific criteria for complex lesions. We also established a National referral centre (NRC) at Llandough with the requisite skills and expertise in complex Endoscopic Mucosal Resection and Dissection (EMR, ESD) where appropriate polyps that met the referral criteria after a Network Multidisciplinary Team meeting discussion (NMDT) could undergo advanced therapy. An NMDT and NRC pilot was established in Oct 2011 to offer the opportunity to access expert opinion and discussion of therapeutic options for Welsh participants of the BCSP. We present our preliminary results.

Methods Referral criteria for complex polyps were agreed based on a composite of site, size, morphology and accessibility. Polyps satisfying the criteria were referred to the NMDT electronically along with relevant images and video. Depending on outcomes of NMDT discussion participants were given the option of accessing local surgery or travelling to the NRC for therapeutic endoscopy. Over a 2 year period, 140 referrals were made from 14 different welsh BCS centres to the NMDT.

Results The various management decisions taken in 126 benign complex polyps and the 14 cancers detected is illustrated in fig 1. Polyps that had incomplete resection (22) often had piecemeal EMR or repeated attempts at EMR at LAC causing failure of lifting in polyps. It is noteworthy that in the first 1 year of NMDT and NRC establishment;16 such cases were referred in contrast to 6 in the subsequent year with most cases in the 1st year needing surgery. This is an encouraging trend as awareness through discussion in the NMDT has streamlined management and decreased the incidence of incomplete resections allowing definitive management in the first instance and reduction in inappropriate referral to surgery for benign disease.

Conclusion Establishing a clinical network for standardised decision making for complex polyps appears to have a significant effect on clinical outcomes.

Disclosure of Interest None Declared.

\section{PTH-066 AN AUDIT TO ASSESS FEASIBILITY AND EFFICACY OF GROUP EDUCATION FOR IRRITABLE BOWEL SYNDROME (IBS) PATIENTS IN THE DELIVERY OF LOW FODMAP (FERMENTABLE OLIGOSACCHARIDES, DISACCHARIDES, MONOSACCHARIDES AND POLYOLS) DIETARY ADVICE}

SK Kinrade, RM Twamley*, L Fell, L Heald, A Healy. Nutrition and Dietetics, University Hospital South Manchester, Manchester, UK

\subsection{6/gutjnl-2014-307263.512}

Introduction A low FODMAP diet has demonstrated symptom improvement in patients with IBS when conducted during individual consultations. ${ }^{1}$ Structured dietetic group education is a well recognised medium for encouraging self-management and promoting confidence in patients with chronic illness ${ }^{2}$ There is a lack of research in the use of low FODMAP dietary intervention in a group setting. This audit was conducted to assess the efficacy and feasibility of providing low FODMAP dietary advice in a group environment.

Methods Data was collected using the 'IBS satisfaction survey'3 which was given to 17 patients with IBS on completion of the 8 week low FODMAP diet. The following question was used to monitor effectiveness of the low FODMAP diet: 'Do you currently have satisfactory relief from your gut symptoms'? This is a closed question, completed anonymously, with a choice response of 'yes' or 'no'. Feasibility of a group format was measured via attendee's feedback and non-attendance (DNA) rate. Feedback was collected using an evaluation questionnaire (6 point Likert scale - very satisfied, satisfied, acceptable, dissatisfied, very dissatisfied, unsure) after the group session.

Results 21 patients attended the initial session. 4 patients (19\%) failed to attend the follow up session. 82\% $(14 / 17)$ of patients who completed the education programme reported satisfactory relief of gut symptoms. $100 \%$ of patients were 'satisfied' or 'very satisfied' with the presentation and group discussion. 94\% were 'satisfied' or 'very satisfied' with the organisation of the group session.

Conclusion Group education for low FODMAP diet therapy is a feasible and effective method for promoting symptom improvement for IBS patients. Group education has the potential to be at least as effective as one-to-one low FODMAP IBS education. Further randomised control studies with large sample sizes are recommended.

\section{REFERENCES}

1 Halmos EP, Power, VA, Shepherd SJ, Gibson PR, Muir JG. Diet Low in FODMAPs Reduces Symptoms of Irritable Bowel Syndrome. Gastroenterology 2014;146(1): pp67-75

2 Keen AJ, Duncan E, McKillop-Smith A, Evans ND, Gold AE. Dose Adjustment for normal eating (DAFNE) in routine clinical practice: who benefits? Diabetes Medicine 2012;29(5):pp670-6

3 Staudacher HM, Whelan K, Irving PM, Lomer MC. Comparison of symptom response following advice for a diet low in fermentable carbohydrates (FODMAPs) versus standard dietary advice in patients with irritable bowel syndrome. Journal of Human Nutrition and Dietetics, 2011;24(5):pp487-95

Disclosure of Interest None Declared.

\section{PTH-067 SETTING UP A HEPATOLOGY ECONSULT SERVICE - BENEFICIAL FOR PATIENTS AND PRIMARY CARE, BUT PERHAPS A HARDSHIP FOR SECONDARY CARE?}

${ }^{1} S$ Moreea*, ${ }^{1}$ VJ Appleby, ${ }^{2 B}$ Smith, ${ }^{~}$ PB Southern, ${ }^{3} \mathrm{~J}$ Connolly. ${ }^{2}$ Digestive Diseases Centre, Bradford Royal Infirmary, Duckworth Lane, UK; ${ }^{2}$ Bradford Royal Infirmary, Duckworth Lane, UK; ${ }^{3}$ Bradford and Airedale Primary Care Trust, Bradford, UK

\subsection{6/gutjpl-2014-307263.513}

Introduction Due to the heavy burden of liver disease, new policies are required to improve information flow between primary care $(\mathrm{PC})$ and secondary care (SC). Outpatient visits are costly to PC, time consuming for patients and not always appropriate. Developing interventions to increase care available in PC is desirable and likely cost effective. We developed a hepatology eConsult $(\mathrm{eC})$ service, allowing PC clinicians to send a referral and share a patient's medical record electronically with SC using a PC database.

Methods Service set up: Discussions between PC and SC identified a need for the service, and thorough review of the current Hepatology service was undertaken, focussing on current and projected working practices, service demands as well as clinicians job plans. Once eC was agreed in principle, a price of $£ 23$ per $\mathrm{eC}$ and a timescale of 7 days for $\mathrm{eC}$ to be completed was agreed with the CCG. A risk assessment of the service was performed and a comprehensive set of guidelines devised for use in PC, ensuring that only appropriate and timely referrals are made. Prior to launching the service, IT systems were updated, and appropriate training delivered to clinicians. To ensure smooth running of $\mathrm{eC}$, user guides and support documents were created and distributed. 
Making and processing a referral using eC: Once a referral has been made in PC, it appears on-line and a hepatology secretary logs the referral, opening the episode of care and informs the designated hepatologist that a referral has been received. The eC takes approximately $15 \mathrm{~min}$ of consultant time to complete but varies depending on case complexity. Once completed, the hepatologist informs the secretary and they log a 'completed episode of care' ensuring the trust is paid for the clinical encounter. Referrals are audited on a 6 monthly basis.

Results Between March 2012 - Oct 2013, 81 eC were completed (12 in months 1-6, 16 in months 7-12, 40 in months 13-18, 13 in months 19-20). A SC appointment was avoided in $78 \%$ of patients $(n=63)$ resulting in a cost saving to PC of $£ 16,443[63 \times \mathrm{eC}(£ 23)=1,449$ vs $63 \times$ new patient referrals $(£ 181)=£ 11,403+1 \mathrm{x}$ follow up/patientn $(£ 103)=£ 6489)$. Median response time for $\mathrm{eC}$ was 2 days, $43 \%$ were completed within the same working day.

Conclusion Hepatology $\mathrm{eC}$ is beneficial for patient care, with specialist advice being provided within one working day in a substantial number of cases, and is clearly cost effective, making eC popular with PC. However, until a more slim-line IT system is developed reducing the number of steps involved in completing an $\mathrm{eC}$, and the cost per eC increased, it appears to be beneficial for all parties except SC.

Disclosure of Interest None Declared.

\section{PTH-068 REDUCING THE OUTPATIENT BURDEN OF 2 WEEK WAIT UPPER GI REFERRALS}

S Singh*, H Dhaliwal, R Keld. Gastroenterology, Wrightington, Wigan and Leigh NHS Trust, Wigan, UK

\subsection{6/gutjnl-2014-307263.514}

Introduction The two week wait (2WW) referral leads to significant burden on outpatient clinics. This delays appointments for patients that may not fit the $2 \mathrm{WW}$ criteria. Only $5 \%$ of patients referred as $2 \mathrm{WW}$ will have an upper GI malignancy and it may not be necessary for all these patients to be reviewed urgently in clinic. Previously at our hospital, most upper GI 2WW referrals were booked an OGD (performed by any available endoscopist) in addition to a clinic appointment on receipt of the referral. In order to streamline the service, in January 2013 patients were triaged to either an OGD or a clinic appointment. The index OGD's are now done on consultant's list (Gastroenterologist and Upper GI Surgeon) with a clinical assessment at their OGD appointment. Further management is protocol based and dependant upon the assessment and OGD findings. The aim of this study was to determine if this change in practice is effective and safe.

Methods Patients referred as a 2WW in January and February 2012 were compared to those referred in January, February, August and September 2013. Only patients triaged directly to OGD were included (77/143 (54\%) in 2012 and 180/291 (62\%) in 2013). 14 patients were excluded from further analysis due to non-attendance.

Results Total cancer detection for all referrals was 8\% in 2012 and $9 \%$ in 2013. In patients selected for a direct OGD referral, 7 upper GI cancers were diagnosed in 2012 and 14 in 2013. After the OGD, $4(5 \%)$ patients in 2012 were immediately discharged back to the GP, compared to $33(20 \%)$ in 2013 ( $\mathrm{p}=$ 0.003). Of those attending clinic post OGD, 9 patients $(13 \%)$ were given a routine appointment in 2012 compared to 50 $(37 \%)$ in $2013(\mathrm{p}=0.0002)$. Comparing the two years, there was a $32 \%$ reduction in the requirement of urgent outpatient appointments (83\% had urgent OPD in 2012 compared to 51\% in 2013, p $=0.0001)$. Of those discharged in 2013, 85\% had documentation of the current symptoms at time of OGD and in $94 \%$, treatment advice was provided to the GP. One patient was discharged after an OGD showing grade B oesophagitis and symptom improvement with PPI. Unfortunately, a re-referral 8 weeks later for worsening symptoms found oesophageal cancer on OGD.

Conclusion The introduction of consultant assessment as a first contact for all OGD 2WW referrals has led to a significant reduction in the requirement of urgent outpatient clinic appointments by one third. Waiting times for all clinic referrals have reduced significantly, amounting to $54 \%$ reduction in the number of patients waiting more than 9 weeks for a first appointment. Cancer detection is comparable to the previous model of care. Patients with ongoing symptoms at the time of endoscopy need follow up. In hindsight the missed cancer should have had an oesophageal biopsy, but this is clinical judgement and we do not believe the new service accounted for this delay.

Disclosure of Interest None Declared.

\section{PTH-069 NURSE TELEPHONE TRIAGED STRAIGHT TO TEST COLONOSCOPY}

${ }^{1}$ A Thapar, 'S Rodney*, 'D Haboubi, '1 Wilson, ${ }^{1} \mathrm{C}$ Bhan, ${ }^{1} \mathrm{M}$ Walshe, ${ }^{2} \mathrm{~J}$ Haddow, ${ }^{1}$ A Oshowo, ${ }^{1} \mathrm{H}$ Mukhtar. ${ }^{1}$ Surgery, Whittington Hospital, London, UK; ${ }^{2}$ National Centre for Bowel Research and Surgical Innovation, Queen Mary University of London, London, UK

\subsection{6/gutjnl-2014-307263.515}

Introduction In 2011 patients referred with suspected colorectal cancer at our institution waited a median of 36 days (IQR2846) for a treatment plan. This resulted in $61 \%$ of colorectal cancerstaking over 31 days to reach a decision to treat. We aimed to reduce the time spent in the diagnostic phase, which was perceived to be the main hold up in the fast track pathway.

Methods A quality improvement approach was employed to change the new pathway from the existing clinic-first service to a straight-to-test service. The new nurse-led telephone triage service confirmed symptoms and assessed fitness for colonoscopy, with higher-risk patients defaulting to flexible sigmoidoscopy or clinic. Results for the first year of the new service are presented.

Results 438 patients were referred between 1/10/2012 and 1/10/ 2013. $222(50 \%)$ went straight to colonoscopy and $136(31 \%)$ to flexible sigmoidoscopy, 46 (11\%) went to clinic, $32(7 \%)$ patients did not attend and data was missing for $2(1 \%)$ patients. Final diagnoses are shown in the attached figure. Colorectal cancer was found in 14/358 patients (4\%). Median time from receipt of referral to first endoscopy was 13 days (IQR 11-20), with 128/348 patients (29\%) waiting more than 14 days. Median time to decision to treat colorectal cancer was 25 days (IQR 20-34) in straight to test patients, a significant reduction compared to 2010-2011 ( $\mathrm{p}=0.01)$, with $5 / 14(36 \%)$ waiting more than 31 days. Median time to first oncological treatment was 40 days (IQR 28-44), with 1/14 (7\%) waiting more than 62 days. 41/66 (62\%) of patients with a normal colonoscopy were discharged directly from endoscopy back to their GP.

Conclusion The new straight to test service was applicable to the majority of new colorectal fast track patients and a high patient uptake was observed. Colorectal cancer was in fact uncommon, which is being fed back to those referring into the 